\title{
Unplanned Extubation in the Neonatal ICU: A Systematic Review, Critical Appraisal, and Evidence-Based Recommendations
}

\author{
Paulo Sérgio Lucas da Silva MD MSc, Maria Eunice Reis MD, Vânia Euzébio Aguiar MD, \\ and Marcelo Cunio Machado Fonseca MD MSc
}

\author{
Introduction \\ Methods \\ Search Strategy \\ Study Selection \\ Statistical Analysis \\ Results \\ Incidence \\ Risk Factors Associated With Unplanned Extubation \\ Immediate Complications After Unplanned Extubation, and Outcome \\ Preventive Measures \\ Endotracheal Tube Fixation \\ Physical Restraints \\ Sedation \\ Nurse-to-Patient Ratio \\ Quality Improvement Programs \\ Discussion \\ Summary
}

\begin{abstract}
OBJECTIVE: To update the state of knowledge on unplanned extubations (UEs) in neonatal ICUs. This review focuses on the following topics: incidence, risk factors, reintubation after UE, outcomes, and prevention. METHODS: The MEDLINE, EMBASE, CINAHL, Scielo, Lilacs, and Cochrane databases were searched for relevant publications from January 1, 1950, through January 30, 2012. Fifteen articles were selected for data abstraction. The search strategy included the following key words: "unplanned extubation," "accidental extubation," "self extubation," "unintentional extubation," "unexpected extubation," "inadvertent extubation," "unintended extubation," "spontaneous extubation," "treatment interference," and "airway accident." Study quality was assessed using the Newcastle-Ottawa scale. Grades of recommendation were assessed according to the Oxford Centre for Evidence-Based Medicine's levels of evidence system. Studies with Newcastle-Ottawa scale score $\geq 5$ that included appropriate statistical analysis were deemed of high methodological quality. RESULTS: The overall mean Newcastle-Ottawa scale score was 3.5. UE rates ranged from 0.14 to $5.3 \mathrm{UEs} / 100$ intubation days, or $1 \%$ to $\mathbf{8 0 . 8 \%}$. Risk factors included restlessness/agitation
\end{abstract}

Drs da Silva and Aguiar are affiliated with the Pediatric Intensive Care Unit, Department of Pediatrics, Hospital Sevidor Público Municipal, São Paulo, Brazil. Dr Reis is affiliated with the Neonatal Intensive Care Unit, Hospital e Maternidade Santa Joana, São Paulo, Brazil. Dr Fonseca is affiliated with the Pediatric Intensive Care Unit, Department of Pediatrics, Universidade Federal de São Paulo (UNIFESP), São Paulo, Brazil.
The authors have disclosed no conflicts of interest.

Correspondence: Paulo Sergio Lucas da Silva MD MSc, Pediatric ICU, Hospital do Servidor Público Municipal, Rua Castro Alves 60, Aclimação, Brazil 01532-000, E-mail: psls.nat@terra.com.br.

DOI: $10.4187 /$ respcare. 02164 
(13-89\%), poor fixation of endotracheal tube (8.5-31\%), tube manipulation at the time of UE $(17-30 \%)$, and performance of a patient procedure at bedside $(27.5-51 \%)$. One study showed that every day on mechanical ventilation increased the UE risk $3 \%$ (relative risk $1.03, P<.001$ ). The association between birth weight/gestational age and $\mathrm{UE}$ is controversial. Reintubation rates ranged from $8.3 \%$ to $100 \%$. There is still a gap of information about strategies addressed to reduce the incidence of UE. The best method of endotracheal tube securement remains a controversial issue. CONCLUSIONS: Despite numerous publications on UE, there are few studies assessing preventive strategies for adverse events and there is a lack of randomized clinical trials. Recommendations are proposed based on the current available literature. Key words: accidental extubation; endotracheal tube; intubation; neonatal ICU; quality improvement; unplanned extubation. [Respir Care 2013;58(7): 1237-1245. (C) 2013 Daedalus Enterprises]

\section{Introduction}

Mechanical ventilation through an endotracheal tube (ETT) is a routine procedure in neonatal ICUs (NICUs), and has contributed to improving critically ill neonates' survival and reducing mortality in NICUs. ${ }^{1}$ Newborns delivered at $<28$ weeks gestational age are more likely to be ventilated than their more mature counterparts. ${ }^{2}$ Therefore, mechanically ventilated neonates are prone to a number of adverse events, such as unplanned extubation (UE). ${ }^{3}$ Of note, UE requiring reintubation is the fourth most common adverse event in NICUs in the United States. ${ }^{3}$ UE is a potentially devastating and costly event, because it may lead to a variety of complications, including serious cardiovascular and respiratory events. ${ }^{4,5} \mathrm{UE}$ is defined as premature removal of the ETT by the patient (deliberate UE) or by staff during nursing and medical care (accidental extubation). ${ }^{6}$ Greater emphasis has been placed on improving the quality of healthcare and patient safety in recent years, and, given the importance of this potentially preventable adverse event, UE rate is monitored by many NICUs as a quality of care metric.

To reduce the UE rate in NICUs there are a variety of distinct approaches and methods, which result in widely different outcomes. Therefore, standardizing the procedures and goals represents an important step toward reducing outcome variability and refining quality improvement processes. A recent systematic review in critically ill children proposed a target benchmark and recommendations on UE prevention encompassing the quality of care components. ${ }^{6}$ However, there is no consensus on strategies for the prevention of this possibly catastrophic event in neonatal care.

The aim of this review was to assess the incidence of UE, its risk factors, the incidence of reintubation after UE, and the outcomes of UE. The present study will also suggest recommendations based on the available evidence to serve as a benchmark of standard care for use in quality improvement programs.

\section{Methods}

\section{Search Strategy}

We searched the United States National Library of Medicine and National Institutes of Health (PubMed), Excerpta Medica (EMBASE), Cumulative Index to Nursing and Allied Health Literature (CINAHL), Cochrane Library, Scientific Electronic Library Online (Scielo), and Literatura Latino Americana em Ciências da Saúde (LILACS) databases for the period spanning from January 1950 to January 2012. The search strategy included the following key words: "unplanned extubation," "accidental extubation," "self extubation," "unintentional extubation," "unexpected extubation," "inadvertent extubation," "unintended extubation," "spontaneous extubation," "treatment interference," and "airway accident." In addition, the reference lists contained in the articles retrieved were checked, and review articles were also included in the search to identify other potentially relevant articles.

\section{Study Selection}

Two authors (PSLS and MER) independently and sequentially reviewed citations, abstracts, and full-text articles to select eligible studies. The titles or abstracts, or both, selected by either author were included in the subsequent step of the selection process. Disagreements were resolved by consensus.

The initial inclusion criteria of the potential studies for this analysis were study populations that comprised mechanically ventilated preterm and term newborns with UEs and outcomes that included UE rate, risk factors associated with UE, reintubation after UE, and strategies to prevent UE. Study designs were cohort, case-control, or crosssectional.

Study quality was evaluated using the Newcastle-Ottawa scale for assessing the quality of non-randomized studies (eg, case-control and cohort studies). ${ }^{7}$ Studies with Newcastle-Ottawa scale score $\geq 5$ that included appropri- 
ate statistical analysis (eg, risk-adjusted or multivariate) were deemed of high methodological quality. Multivariate analysis or other acceptable methods of adjusting for risk were required to reduce confounding. Two reviewers (PSLS and MER) independently rated each study, and variations in ratings were reconciled via discussion. Grades of recommendation were assessed according to the Oxford Centre for Evidence-Based Medicine's levels of evidence system. ${ }^{8}$

\section{Statistical Analysis}

We used descriptive statistics according to the variable characteristics. The medians and interquartile range (25th and 75th percentiles) are presented for continuous variables. Pareto charts present a summary of the data gleaned in this literature review.

\section{Results}

The combined computerized and bibliographic literature search yielded 34,105 potentially relevant studies, of which 192 studies were identified for more detailed review. Fifteen of these studies met the inclusion criteria. ${ }^{1,3,5,9-20}$ Of the 15 studies reviewed, 11 were prospective cohort studies. ${ }^{3,10-13,15-20} 3$ were retrospective cohort studies, ${ }^{1,9,14}$ and 1 was a retrospective and prospective cohort study. ${ }^{5}$ All the studies were in English. A total of 12 studies $^{3,5,9,10,12-20}$ were considered of low methodological quality, and 3 of high methodological quality. ${ }^{1,11,18}$ The overall mean Newcastle-Ottawa scale score was 3.5.

\section{Incidence}

Studies conducted over the last 30 years reported UE rates ranging from $0.14 \mathrm{UEs} / 100$ intubation days ${ }^{9}$ to 5.3 UEs/100 intubation days ${ }^{18}$ (median 1.98, IQR 0.913.8 ), or $1 \%$ to $80.8 \% 15$ (median $18.2 \%$, IQR $5.37-45.6 \%$ ). Nevertheless, this incidence has not changed over the last 5 years, with reported rates ranging from 0.56 UEs/100 intubation days ${ }^{19}$ to $5.3 \mathrm{UEs} / 100$ intubation days ${ }^{18}$ (median 1.98, IQR $1.06-4.22$ ) or from $1.28 \%{ }^{19}$ to $58 \%{ }^{18}$ (median $8.4 \%$, IQR $3.7-51.6 \%$ ).

\section{Risk Factors Associated With Unplanned Extubation}

While one study showed a higher incidence of UE in infants older than 34 weeks gestational age (89\%), ${ }^{19} 2$ studies found no association between gestational age and UE. ${ }^{1,17}$ With regard to association between UE and weight, the studies are also controversial. Brown ${ }^{11}$ found that UE rate was higher in neonates $<1,500 \mathrm{~g}$ than in neonates $>1,500 \mathrm{~g}(42 \%$ vs $23 \%, P<.04)$. Likewise, Horimoto et $\mathrm{al}^{14}$ found a higher UE rate in infants $<2,500 \mathrm{~g}(87 \%)$. On the other hand, 3 studies ${ }^{1,12,17}$ showed no difference in weight between UE infants and controls.

Eleven studies reported the circumstances of when UE occurred. ${ }^{1,5,11-13,15-20}$ Seven studies ${ }^{1,5,11-13,17,19}$ identified that restlessness/agitation occurred in between $13 \% 5$ and $89 \%{ }^{19}$ of all patients who had UE (median $25 \%$, IQR $16.6-$ $57.2 \%$ ), while one study reported that self-extubation (ETT coughed out or pulled out) constituted $62 \%$ of the total of UEs. ${ }^{15}$ Risk factors associated with UE include poor fixation (loose or wet tape) and ETT manipulation (suctioning, retaping of ETT, and unsupported ventilator tubing). The frequency of UEs associated with poor fixation ranged from $8.5 \%^{11}$ to $31 \%^{5}$ of the total of UEs (median $31 \%$, IQR 14.9-37.5\%), whereas ETT manipulation at the time of UE ranged from $17 \%^{16}$ to $30 \%{ }^{15}$ (median 26.5, IQR 21-29\%) of all patients who had UE. UEs due to a patient procedure at bedside ranged from $27.5 \%{ }^{11}$ to $51 \%{ }^{17}$ of all UE events (median 46.1, IQR 35.4-50\%). Two studies reported UE during kangaroo care $\left(8 \%{ }^{1}\right.$ and $21 \% 17$ of the total of UE). The use of physical restraints was assessed in 2 studies. ${ }^{12,13}$ The percentage of patients under physical restraint at the time of UE ranged from $35 \%{ }^{12}$ to $87 \%^{13}$ of the total of UEs in these studies.

Other contributing factors, among the total of patients experiencing UEs, included previous UE (47\%), ${ }^{12}$ weaning stage from mechanical ventilation $(44.4 \%),{ }^{19}$ and day shift $(51 \%) .{ }^{12}$ Also, Brown ${ }^{11}$ found that the most significant factors predicting UE were the length of time intubated $(P<.001)$ and the taping method $(P<.02)$.

Nevertheless, only 2 studies assessed the risk factors for UE employing multivariate analysis. ${ }^{18,20}$ Carvalho et al ${ }^{18}$ showed that assisted ventilation duration was the only independent predictor of UE. They analyzed gestational age, birth weight, sex, use of analgesia/sedation, intubation route, and total number of patient-days ventilated/month. Every day on mechanical ventilation increased UE risk 3\% (relative risk 1.03, $P<.001$ ), and an assisted ventilation time of 10.5 days had an accuracy of $80 \%$ in identifying the occurrence of UE.

The number of studies that assessed each risk factor is presented in Figure 1.

\section{Immediate Complications After Unplanned Extubation, and Outcome}

Two studies ${ }^{12,14}$ reported hemodynamic complications immediately after UE. In relation to the total of patients who experienced UEs, the frequencies of bradycardia were $39 \%{ }^{12}$ and $46 \%,{ }^{14}$ respectively, and the percentage of neonates requiring cardiopulmonary resuscitation were $5 \%{ }^{12}$ and $13 \% 14$ in these studies.

Six studies $1,3,5,10,15,18$ reported reintubation rates ranging from $8.3 \%^{3}$ to $100 \%^{9}$ in patients who had UE (median 


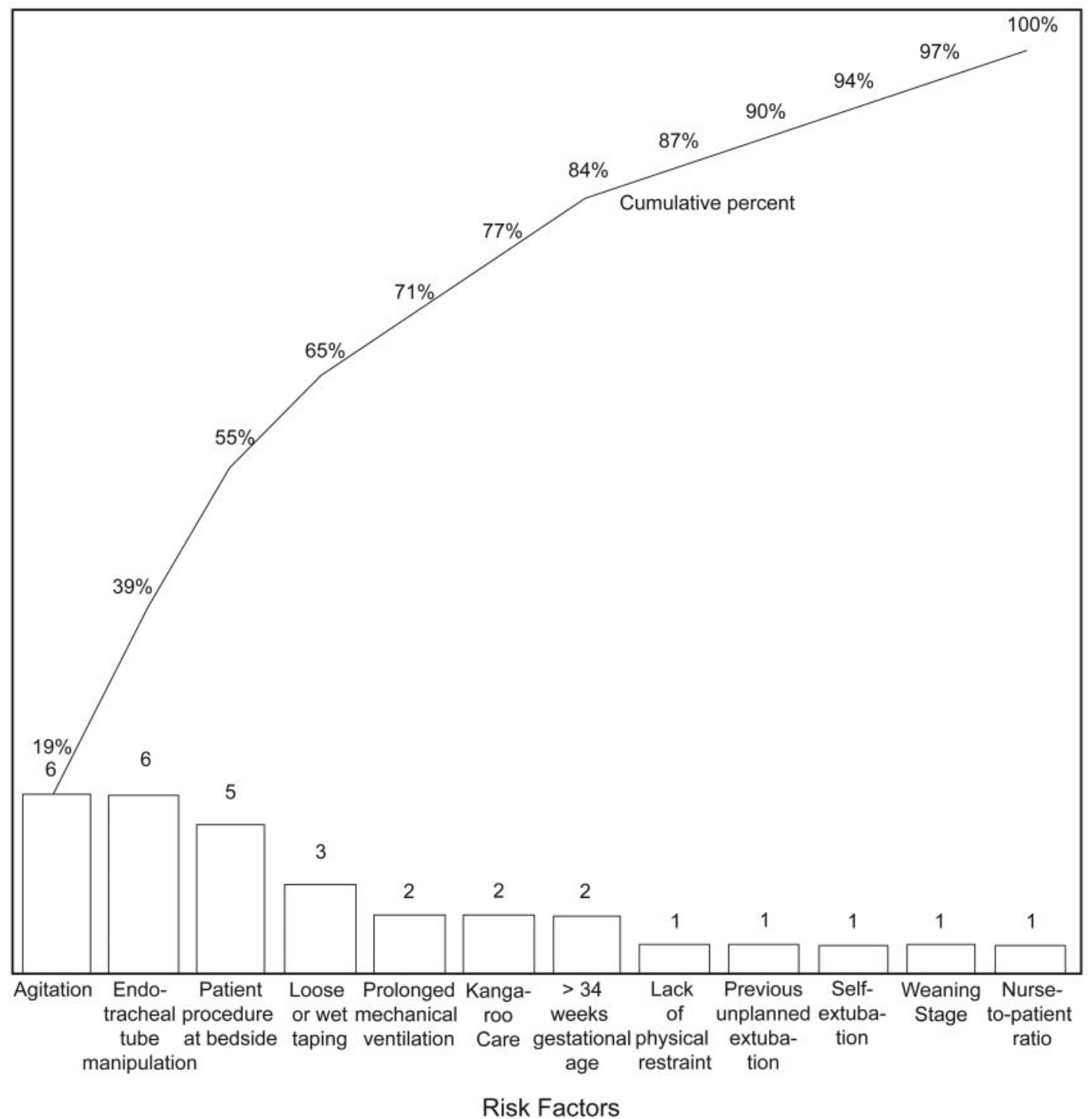

Fig. 1. Pareto chart of reported risk factors for unplanned extubations in the neonatal ICU.

58\%, IQR 17.9-69.3\%). We did not find neonatal studies describing predictors for reintubation in neonates who experienced UE.

Only one study assessed the impact of UE on outcome in NICU patients. ${ }^{1}$ Veldman et $\mathrm{al}^{1}$ reported longer mechanical ventilation in UE neonates $(345 \mathrm{~h}$ vs $52 \mathrm{~h}$, difference not significant) and longer NICU stay ( $51 \mathrm{~d}$ vs $9 \mathrm{~d}$, $P=.008)$, compared to controls.

\section{Preventive Measures}

Although several studies have been published over the last 30 years on UE in NICU patients, only a few studies have assessed UE preventive measures.5,16,17,19 Three of these studies reported the use of alternative methods for securing ETTs, ${ }^{16,17,19}$ while 2 other studies described the impact of a quality improvement program. ${ }^{5,19}$
Likewise, there is a paucity of studies evaluating the impact of specific measures such as the use of ETT securement, physical restraints, sedation, and ventilationweaning protocols.

In Figure 2 we present the number of studies that assessed each preventive measure. Eighty-three percent of the included studies appraised ETT fixation, weaning and extubation protocols, nurse-to-patient ratio suitability, and attention to the infant with a previous UE. Likewise, in Figure 3 are the main interventions studied to prevent UE and their frequencies.

\section{Endotracheal Tube Fixation}

Nine studies described the method utilized for securing ETTs. ${ }^{15,9-12,15-17}$ Although the techniques for stabilizing 


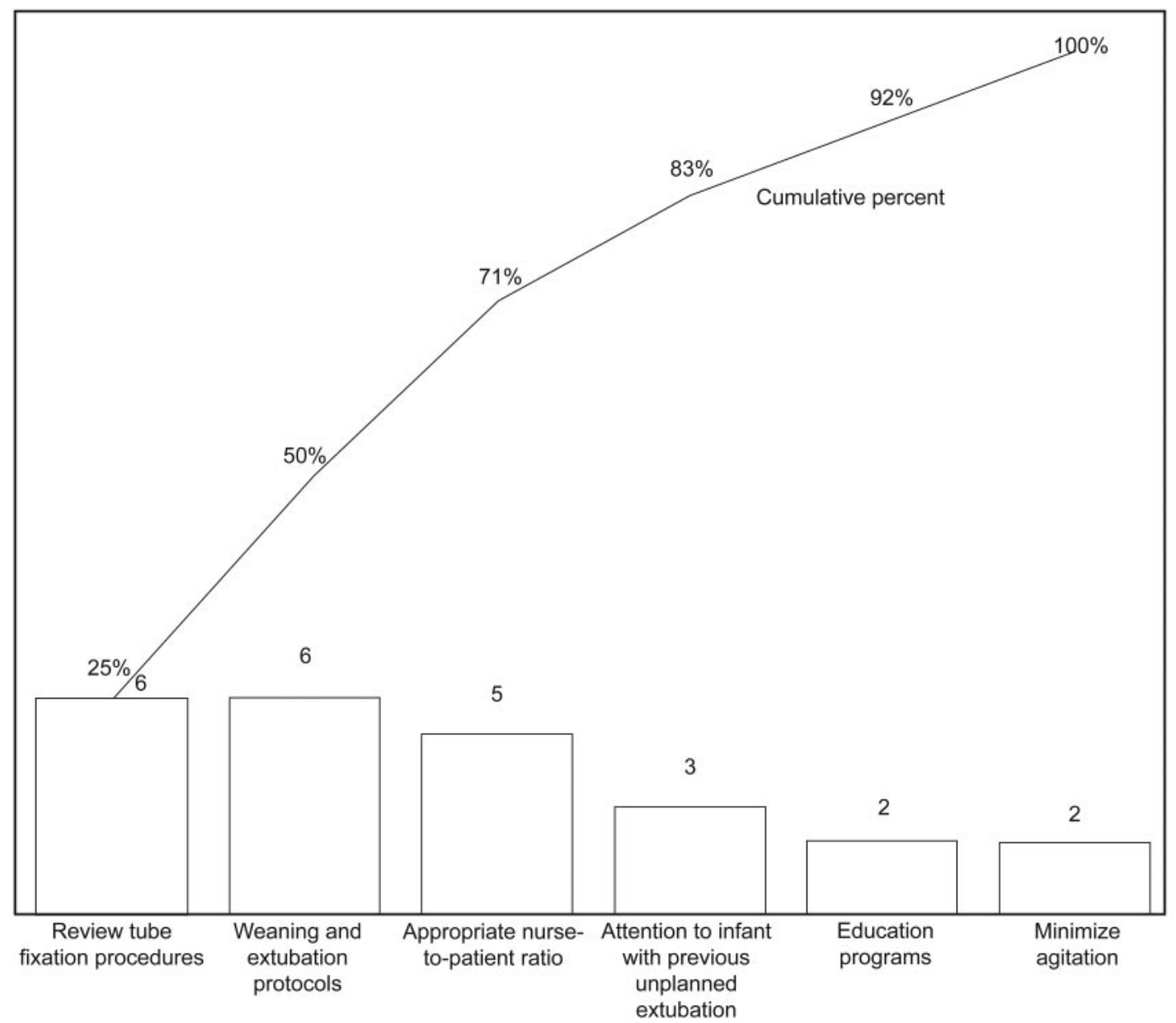

Preventive Measures

Fig. 2. Pareto chart of reported preventive measures for unplanned extubations in the neonatal ICU.

ETTs differed across studies, 4 of them ${ }^{9-12}$ were based on the method described by Gregory. ${ }^{21}$ Other techniques for securing the ETT included the umbilical clamp method, ,5,17 2 strips of adhesive Y-shaped tape, ${ }^{1,16}$ the pectin-based skin barrier adhesive tape ${ }^{15}$ described by Lund et al, ${ }^{22}$ and the Logan Bow (nuchal arch). ${ }^{16}$ The conventional taping method involves 2 Y-shaped or $\mathrm{H}$-shaped adhesive tape strips.

Volsko and Chatburn ${ }^{16}$ found that the use of the Logan Bow to secure ETTs reduced the UE rate of infants weighing $\leq 1,500 \mathrm{~g}$, when compared to the conventional method (21.3\% vs $58.7 \%, P<.001)$. Two studies employing the umbilical clamp technique for endotracheal stabilization demonstrated a reduction of UE rate by approximately 40\%..$^{5,17}$ DeJonge and White ${ }^{17}$ described a reduction of UE rate from 3.2 to $1.8 \mathrm{UEs} / 100$ intubation days $(P=.001)$ after changing the traditional $\mathrm{H}$-shaped taping method to the umbilical clamp taping. Loughead et $\mathrm{al}^{5}$ reported an immediate decrease in UE rate, related to a change to the umbilical clamp taping method, from 4.2 to $2.5 \mathrm{UEs} / 100$ ventilator days. Two studies reported that nurses and respiratory therapists evaluated ETT security every 2 hours $^{13}$ or 3 hours. ${ }^{10}$

\section{Physical Restraints}

There were conflicting data regarding the influence of physical restraints and UEs. In fact, few studies ${ }^{11-13,15}$ mentioned the use of restraints, and only 2 of them provided complete information..$^{11,13}$ Two studies ${ }^{12,15}$ found that the use of limb restraints did not differ between in infants who did and did not extubate. In contrast, 2 other studies showed the role of restraints for UE prevention. Little et $\mathrm{al}^{13}$ showed that the lack of 2 or more points of restraint contributed to UE in 58\% of all patients who had UE. Brown ${ }^{11}$ reported that head restraint may benefit infants who are more agitated. However, methodological flaws hamper the interpretation of these results. 


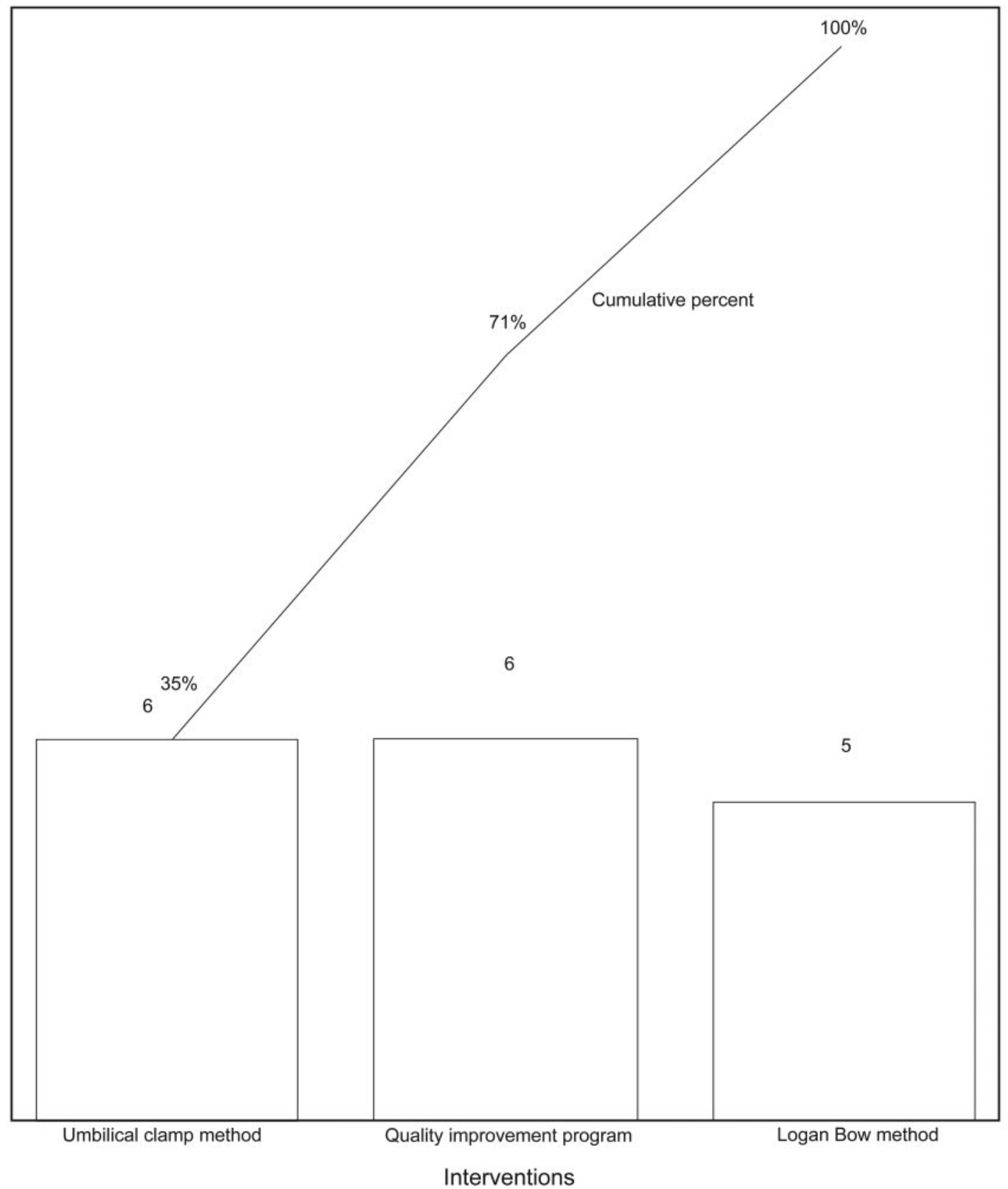

Fig. 3. Pareto chart of reported interventions for unplanned extubations in the neonatal ICU.

\section{Sedation}

There are no studies in NICU patients properly assessing the use of sedatives to prevent UE. Two studies ${ }^{12,15}$ reported that the use of sedatives was not associated with an increased incidence of UE. On the other hand, 2 authors ${ }^{1,13}$ showed a high UE occurrence in patients who had not previously received sedative agents. Furthermore, Carvalho et $\mathrm{al}^{18}$ found that UE rates were higher in newborns without sedation/analgesia; however, this variable was not associated with $\mathrm{UE}(P=.86)$, probably due to the limited number of patients without sedation (12\% of the total ventilated patients). Only one study ${ }^{1}$ mentioned the sedative/ analgesic agents (morphine, benzodiazepines, or phenobarbital) administered in the NICU. Again, the serious methodological flaws of these studies do not allow for any consistent conclusion.

\section{Nurse-to-Patient Ratio}

Three studies ${ }^{1,12,15}$ did not find a correlation between UE and nursing work load. Kleiber and Hummel ${ }^{12}$ found 
that a professional was at the bedside during $75 \%$ of the events, while Franck et al ${ }^{15}$ showed no association with nurse-to-patient ratio. In addition, Veldman et $\mathrm{al}^{1}$ reported that nursing staff experience and the work load of nurses were not reasons for UE. On the other hand, a study by Lamy Filho et $\mathrm{al}^{20}$ demonstrated that the larger the number-of-newborns-classified-by-care-demand (number of newborns present during each shift by the number of professionals in each category) per nurse and nursing technician, the more likely the occurrence of intermediate adverse events linked to mechanical ventilation. A numberof-newborns-classified-by-care-demand $>22$ per nurse (relative risk 2.86) and $>4.8$ per nursing technician (relative risk 3.41) was associated with a higher prevalence of intermediate adverse events.

\section{Quality Improvement Programs}

Continuous quality improvement programs involved multiple interventions and focused mainly on data collection tool elements, identification of risk factors leading to UE events, standardization of procedures, and care practices related to the method of securing the ETT, staff education, and early extubation with nasal CPAP support. We identified 3 studies reporting the impact of a quality improvement program on UE occurrence. ${ }^{5,18,19}$

Loughead et $\mathrm{al}^{5}$ showed that the primary reason for the improvement in the UE rate was a process improvement project that also included a change of the method of ETT stabilization. The improvement quality project comprised 3 different phases. Consistent with Plan-Do-Check-Act quality improvement cycles during the first period (baseline), the authors performed data collection and review, and inconsistency in taping methods was identified as the cause of most UEs. In the second period (intervention 1) the authors changed to a single, consistent, conventional taping methodology (bilateral Y-shaped tape strips). Throughout this period the UE rate improved but still remained steadily above the target rate. In the third period (intervention 2) the authors instituted the cord clamp method for ETT fixation. The authors then were able to demonstrate a significant and sustained overall reduction in UE, from 4.8 to $0.9 \mathrm{UEs} / 100$ ventilator days. This improvement was found in all weight groups. Nevertheless, the smaller the birth weight, the greater the degree of improvement in the incidence of UE. Thus, infants with birth weight $>2,500 \mathrm{~g}$ experienced a $53 \%$ reduction in UE rate, while the groups weighing $1,000-2,500 \mathrm{~g}$ and $<1,000 \mathrm{~g}$ had $77 \%$ and $86 \%$ reductions in UE rate, respectively.

Ligi et $\mathrm{al}^{19}$ observed that the UE rate increased from $0.56 \mathrm{UEs} / 100$ intubation days in the baseline period to 1.55 UEs/100 intubation days after implementation of a quality improvement program $(P=.03)$. However, according to the authors, no conclusion should be drawn from this finding, because of the negative effect of major changes during the study, such as the banishment of tincture of benzoin, a reduction in the use of bilateral Yshaped tape strips in ETT fixation, and a high turnover of caregivers.

Carvalho et $\mathrm{al}^{18}$ reported an intervention program to reduce the occurrence of UE, which included staff education, ETT fixation and suctioning, standardization of care, and analgesia and sedation. According to the authors, the mean UE rate reduced from 6.5 to 4.4 UEs/100 intubation days.

\section{Discussion}

UE is a common event in the NICU and may be associated with serious conditions such as bronchospasm, aspiration pneumonia, hypotension, arrhythmias, cardiorespiratory arrest, and even death. ${ }^{4}$ Moreover, UE often leads to emergency endotracheal reintubation in a less controlled scenario. ${ }^{5}$ Repeated intubations, especially those performed in emergencies, increase the risk of laryngeal or tracheal injury and scarring, pulmonary injury from excessive ventilation, intraventricular hemorrhage, ${ }^{5}$ and physiologic changes such as hypoxemia, hypercarbia, increased arterial pressure, and increased intracranial pressure. ${ }^{5}$

Our literature review showed that there are few studies assessing UE in neonatal ICUs. It also revealed that highquality studies are scarce, and only a few of them assessed the effectiveness of strategies to reduce the incidence of UE. Furthermore, the vast majority of the available studies simply report the incidence and risk factors associated with UE, present methodological flaws, and lack proper statistical analysis. In addition, the search yielded no casecontrol studies, relevant systematic reviews, or controlled, randomized clinical trials.

The incidence of UE is expressed as a percentage (number of UEs divided by the number of ventilated patients) or as the number of UEs per 100 intubation days. The latter measure incorporates the concept of days as an exposure factor for event occurrence, so it is more suitable, as it allows comparison among different NICUs. The overall incidence of UE in NICU infants was higher than that reported for the pediatric (0.11-2.7 UEs/100 intubation days $)^{6}$ and adult (0.10-3.62 UEs/100 intubation days $)^{23,24}$ populations. There is evident room for improvement of these NICU results, and this is a particularly timely topic in light of the growing accreditation and regulatory standards aimed at improving patient safety in acute and critical care settings.

We used Pareto charting as a basic quality tool to summarize the main findings of this study. Pareto charts are useful for focusing on the areas of a process that will have the greatest impact in achieving the desired results. Figures 1 and 2 detail risk factors and preventive measures 
Table. Recommendations

\begin{tabular}{|c|c|c|c|}
\hline Area of Study & Recommendations & Comments & Authors' Suggestions \\
\hline Incidence (benchmarking) & $\begin{array}{l}\text { Report incidence in terms of number } \\
\text { of unplanned extubations per } 100 \\
\text { mechanical ventilation days } \\
\text { (recommendation grade C). } \\
\text { Benchmark of }<1 \text { unplanned } \\
\text { extubation/100 intubation days as } \\
\text { a target for quality improvement. } \\
\text { (recommendation grade D). }\end{array}$ & $\begin{array}{l}\text { Although the Institute for Healthcare } \\
\text { Improvement recommends that the } \\
\text { goals for safety measures, such as } \\
\text { ventilator-associated pneumonia } \\
\text { and central line-associated } \\
\text { bloodstream infection, should be } \\
\text { zero, this target does not seem } \\
\text { achievable, and therefore appears } \\
\text { unrealistic for an unplanned } \\
\text { extubation benchmark. }{ }^{27} \\
\text { Despite the limitations in } \\
\text { establishing this objective, this } \\
\text { benchmarking proved to be the } \\
\text { most suitable for use as a } \\
\text { benchmark. }\end{array}$ & \\
\hline $\begin{array}{l}\text { Endotracheal tube securement } \\
\text { and stabilization }\end{array}$ & None & & $\begin{array}{l}\text { Standardizing endotracheal tube } \\
\text { securement procedures and constant } \\
\text { vigilance on tube securement appear } \\
\text { to be the key components for } \\
\text { stabilizing endotracheal tubes } \\
\text { (recommendation grade C) } \text { ) }^{1,11,12}\end{array}$ \\
\hline Sedation and analgesia & & $\begin{array}{l}\text { To be addressed in randomized } \\
\text { controlled trials }\end{array}$ & None \\
\hline Weaning & None & & $\begin{array}{l}\text { Implementation of protocols that help } \\
\text { identify patients ready for extubation } \\
\text { (recommendation grade D). } \\
\text { Noninvasive ventilation can be effective } \\
\text { in preventing extubation failure in } \\
\text { preterm infants, following a period of } \\
\text { endotracheal intubation } \\
\text { (recommendation grade B). } \\
\text { Infants, mostly term neonates, who have } \\
\text { initiated the weaning process should } \\
\text { be assessed for early extubation } \\
\text { (recommendation grade D). }\end{array}$ \\
\hline Physical restraints & None & & \\
\hline Nursing staff & None & & $\begin{array}{l}\text { Maintaining an adequate nurse/patient } \\
\text { ratio, continuing education, training, } \\
\text { and updating standard procedures for } \\
\text { the care of intubated patients may } \\
\text { help prevent unplanned extubation } \\
\text { (recommendation grade C). }\end{array}$ \\
\hline
\end{tabular}

reported in the literature review. It was clear that agitation, ETT fixation, patient procedure at bedside, loose/wet taping, and prolonged mechanical ventilation comprised the few important risk factors for UE, forming $70 \%$ of all reported reasons (see Fig. 1).

On the one hand, agitation was one of the most assessed risk factors (20\% of the studies). Sedation as a method to minimize agitation was not as studied. On the other hand, there is evidence that a sedation/analgesia protocol significantly reduces the incidence of UE in both pediatric ${ }^{25}$ and adult $^{26}$ patients.

Still, in the context of the main risk factors, we also found that ETT manipulation and loose or wet taping com- prised one third of the studies, and that ETT securing techniques, like the umbilical clamp and Logan Bow methods, included $64 \%$ of the most studied interventions (see Fig. 3).

Of note, the finding that a large proportion of infants successfully tolerated UE suggests that many patients are kept on mechanical ventilation longer than necessary Hence, early extubation intended to reduce the duration of mechanical ventilation, especially once the weaning process has commenced, should be taken into account as another strategy to reduce UE. ${ }^{18}$

$\mathrm{UE}$ is a quality measure involving multifactorial causes. Thus, it is plausible to assume that interventions on quality 
factors may be important in reducing UEs. One third of the included studies addressed the use of quality improvement studies in order to reduce UE, because the development of appropriate data tracking tools, data collection, real-time reporting to all caregivers, peer champions' education, and reinforcement of best practices are key components of clinical process improvement. ${ }^{5}$ Thus, we suggest some recommendations for preventing UEs based on what is available in the literature (Table). ${ }^{8}$

\section{Summary}

In summary, we reported a review of the literature assessing the main topics involved in UE and proposed a set of recommendations for UE prevention based on the available studies. The limitations in suggesting recommendations on major care activities, such as the ideal method for securing ETTs, sedation/analgesia protocols, and weaning protocols, are due to the studies' methodological flaws and the limited available evidence. The small number of publications assessing the use of preventive measures, in addition to the lack of randomized clinical trials, underscores the need for future studies aiming to prevent UE events. These recommendations provide a first step forward to improving the science behind the prevention of UEs.

\section{REFERENCES}

1. Veldman A, Trautschold T, Weiss K, Fischer D, Bauer K. Characteristics and outcome of unplanned extubation in ventilated preterm and term newborns on a neonatal intensive care unit. Paediatr Anaesth 2006;16(9):968-973.

2. Bellu R, de Waal K, Zanini R. Opioids for neonates receiving mechanical ventilation: a systematic review and meta-analysis. Arch Dis Child Fetal Neonatal Ed 2010;95(4):F241-F251.

3. Sharek PJ, Horbar JD, Mason W, Bisarya H, Thurm CW, Suresh G, et al. Adverse events in the neonatal intensive care unit: development, testing, and findings of an NICU-focused trigger tool to identify harm in North American NICUs. Pediatrics 2006;118(4):13321340.

4. Bouza C, Garcia E, Diaz M, Segovia E, Rodriguez I. Unplanned extubation in orally intubated medical patients in the intensive care unit: a prospective cohort study. Heart Lung 2007;36(4):270-276.

5. Loughead JL, Brennan RA, DeJuilio P, Camposeo V, Wengert J, Cooke D. Reducing accidental extubation in neonates. Jt Comm J Qual Patient Saf 2008;34(3):164-170, 125.

6. Lucas da Silva PS, de Carvalho WB. Unplanned extubation in pediatric critically ill patients: a systematic review and best practice recommendations. Pediatr Crit Care Med 2010;11(2):287-294.

7. Wells GA, Shea B, O'Connell D, Peterson J, Welch V, Losos M, Tugwell P. The Newcastle-Ottawa Scale (NOS) for assessing the quality if nonrandomized studies in meta-analyses. http://www.ohri. ca/programs/clinical_epidemiology/oxford.htm. Accessed April 18, 2013.

8. Oxford Centre for Evidence-based Medicine. Levels of evidence and grades of recommendation, March 2009. http://www.cebm.net/ index.aspx?o=1025. Accessed April 18, 2013.
9. Conner GH, Maisels MJ. Orotracheal intubation in the newborn. Laryngoscope 1977;87(1):87-91.

10. McMillan DD, Rademaker AW, Buchan KA, Reid A, Machin G, Sauve RS. Benefits of orotracheal and nasotracheal intubation in neonates requiring ventilatory assistance. Pediatrics 1986;77(1):3944.

11. Brown MS. Prevention of accidental extubation in newborns. Am J Dis Child 1988;142(11):1240-1243.

12. Kleiber C, Hummel PA. Factors related to spontaneous endotracheal extubation in the neonate. Pediatr Nurs 1989;15(4):347-351.

13. Little LA, Koenig JC Jr, Newth CJ. Factors affecting accidental extubations in neonatal and pediatric intensive care patients. Crit Care Med 1990;18(2):163-165.

14. Horimoto Y, Tomie H, Hanzawa K, Nishida Y. Accidental extubations during respiratory management in a children's hospital. J Anesth 1991;5(2):142-145.

15. Franck LS, Vaughan B, Wallace J. Extubation and reintubation in the NICU: identifying opportunities to improve care. Pediatr Nurs 1992; 18(3):267-270.

16. Volsko TCR. Comparison of two methods for securing the endotracheal tube in neonates. Respir Care 1997;42(3):288-291.

17. DeJonge MH, White M. A comparison of two methods of oral endotracheal tube stabilization in neonatal patients. J Perinatol 1998; 18(6 Pt 1):463-465.

18. Carvalho FL, Mezzacappa MA, Calil R, Machado Hda C. Incidence and risk factors of accidental extubation in a neonatal intensive care unit. J Pediatr (Rio J) 2010;86(3):189-195.

19. Ligi I, Millet V, Sartor C, Jouve E, Tardieu S, Sambuc R, et al. Iatrogenic events in neonates: beneficial effects of prevention strategies and continuous monitoring. Pediatrics 2010;126(6):e1461e1468.

20. Lamy Filho F, da Silva AA, Lopes JM, Lamy ZC, Simoes VM, Dos Santos AM. Staff workload and adverse events during mechanical ventilation in neonatal intensive care units. J Pediatr (Rio J) 2011; 87(6):487-492.

21. Gregory GA. Respiratory care of newborn infants. Pediatr Clin North Am 1972;19(2):311-324.

22. Lund C, Kuller JM, Tobin C, Lefrak L, Franck LS. Evaluation of a pectin-based barrier under tape to protect neonatal skin. J Obstet Gynecol Neonatal Nurs 1986;15(1):39-44.

23. Castelloes TM, da Silva LD. Guideline for nursing care in the prevention of accidental extubation. Rev Bras Enferm 2007;60(1):106109.

24. Ayllon Garrido N, Rodriguez Borrajo MJ, Soleto Paredes G, Latorre Garcia PM. Unplanned extubations in patients in the ventilator weaning phase in the intensive care unit: incidence and risk factors. Enferm Clin 2009;19(4):210-214.

25. Popernack ML, Thomas NJ, Lucking SE. Decreasing unplanned extubations: utilization of the Penn State Children's Hospital Sedation Algorithm. Pediatr Crit Care Med 2004;5(1):58-62.

26. Powers J. A sedation protocol for preventing patient self-extubation. Dimens Crit Care Nurs 1999;18(2):30-34.

27. Berwick D. The 100,000K Lives Campaign: lessons learned from national mobilization. Plenary discussion. Institute for Healthcare Improvement, 2nd Annual Summit on Redesigning Care, Atlanta, June 2006.

28. Davis PG, Henderson-Smart DJ. Nasal continuous positive airways pressure immediately after extubation for preventing morbidity in preterm infants. Cochrane Database Syst Rev 2003(2):CD000143. 